\section{Chemical and Biochemical Thermodynamics Reunification}

by A. Sabatini, M. Borsari, L.M. Raff, W.R. Cannon, and S. lotti

Chemical equations are written in terms of specific ionic and elemental species and balance elements and charge, whereas biochemical equations are written in terms of reactants that often consist of species in equilibrium with each other and do not balance elements that are assumed fixed, such as hydrogen and magnesium at constant $\mathrm{pH}$ and $\mathrm{pMg}$. When $\mathrm{pH}$ and $\mathrm{pMg}$ are specified, the conditional equilibrium constant $K$ ' for a biochemical reaction is written in terms of sums of species and can be used to calculate a standard Gibbs energy of reaction $\Delta_{\mathrm{r}} G^{\prime 0}$ A chemical equation, as an example, is:

$$
\begin{gathered}
\mathrm{MgATP}^{2-}+\mathrm{H}_{2} \mathrm{O}=\mathrm{MgADP}^{-}+\mathrm{HPO}_{4}^{2-}+\mathrm{H}^{+} \\
K=\frac{\left[\mathrm{MgADP}^{-}\right]\left[\mathrm{HPO}_{4}^{2-}\right]\left[\mathrm{H}^{+}\right]}{\left[\mathrm{MgATP}^{2-}\right]}
\end{gathered}
$$

whereas the biochemical equation is

$$
\begin{gathered}
\mathrm{ATP}+\mathrm{H}_{2} \mathrm{O}=\mathrm{ADP}+\mathrm{P}_{\mathrm{i}} . \\
K^{\prime}=\frac{[\mathrm{ADP}]\left[\mathrm{P}_{i}\right]}{[\mathrm{ATP}]}
\end{gathered}
$$

In the "Recommendations for nomenclature and tables in biochemical thermodynamics" the IUPACIUBMB Joint Commission on Biochemical Nomenclature (JCBN) [1] states: "When $\mathrm{pH}$ and pMg are specified, a whole new set of transformed thermodynamic properties come into play. These properties are different from the usual Gibbs energy $G$, enthalpy $H$, and entropy $S$ and they are referred to as the transformed Gibbs energy $G^{\prime}$, transformed enthalpy $H^{\prime}$ ', transformed entropy $S$ '." As a consequence, two categories of thermodynamics based on different concepts and different formalisms have been established: i) chemical thermodynamics that employs conventional thermodynamic quantities to deal with chemical reactions; ii) biochemical thermodynamics that employs transformed thermodynamic quantities to deal with biochemical reactions.

In his works, Alberty [2,3] has shown how to obtain the transformed thermodynamic quantities $\Delta_{\mathrm{r}} G^{\prime 0}, \Delta_{\mathrm{r}} H^{\prime 0}$, and $\Delta_{\mathrm{r}} S^{\prime 0}$ from $\Delta_{\mathrm{f}} G^{0}, \Delta_{\mathrm{f}} H^{0}$, and $\Delta_{\mathrm{f}} S^{0}$, and of the specific chemical species. According to Alberty $\Delta_{\mathrm{r}} G$ and $\Delta_{\mathrm{r}} G^{\prime}$ provide Gibbs energy of reaction of chemical and biochemical reaction, respectively. In literature numerous

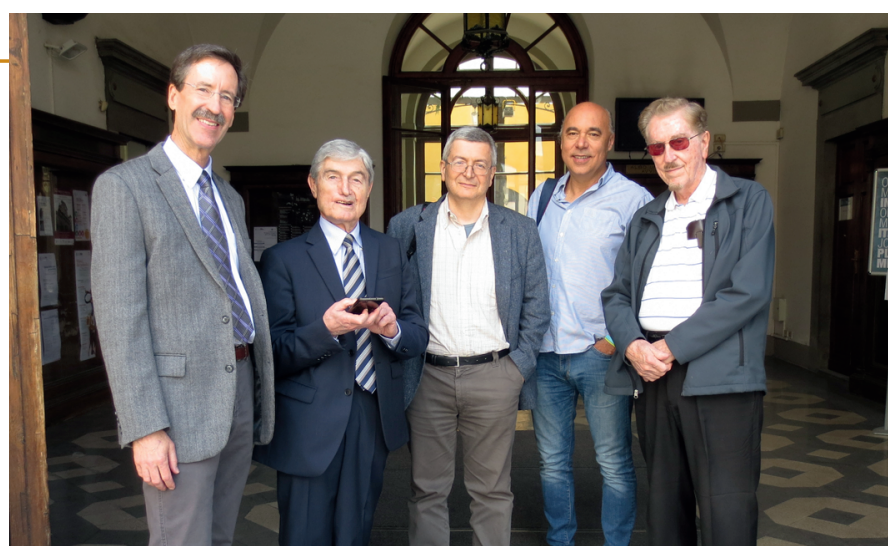

The task group met in Florence, Italy on 23 May 2018; from left: William Cannon, Antonio Sabatini, Marco Borsari, Stefano lotti, Raff Lionel.

papers treating the thermodynamics of a variety of biochemical reactions assert that the two quantities are not the same. However, it has been shown that $\Delta_{\mathrm{r}} \mathrm{G}=\Delta_{\mathrm{r}} \mathrm{G}^{\prime}$ regardless of the reaction involved [4]. This fact has not been sufficiently divulged, and it is still not generally understood by the scientific community. It must be underlined that this equality is restricted to Gibbs energy changes and does not apply to enthalpy or entropy changes.

Moreover, it has been subsequently shown [5] that the "transformed" thermodynamic quantities can be obtained without performing any "transformation" simply balancing a chemical reaction whose reactants and products are the specific species involved in the biochemical reaction.

In a document prepared by the task group including A. Sabatini, M. Borsari, L.M. Raff, W.R. Cannon, and S. Iotti, and available online from the project webpage, the two different methods are analyzed in details. UItimately, through this IUPAC project 2017-021-2-100, the task group plans to show that the use of these methods allows the two worlds of chemical and biochemical thermodynamics, which so far have been treated separately, can be reunified within the same thermodynamic framework.

\section{References:}

1. R. A. Alberty, Pure Appl. Chem., 66, 1641-1666 (1994).

2. R. A. Alberty, Biophys. Chem., 42, 117-131 (1992).

3. R. A. Alberty, Biophys. Chem., 43, 239-254 (1992).

4. S. Iotti, A. Sabatini, and A, Vacca, J. Phys. Chem. B, 114, 1985-1993 (2010).

5. A. Sabatini, A. Vacca, and S. Iotti, PLoS ONE । www.plosone.org, 7, e29529 (2012).

For more information and comments, contact Task Group Chair, Stefano lotti <stefano.iotti@unibo.it>.

www.iupac.org/project/2017-021-2-100 